\title{
Toward on-board Microchip Synthesis of CdSe vs PbSe Nanocrystalline Quantum Dots as a Spectral Decoy for Protecting Space Assets
}

\author{
Job Nijhuis ${ }^{\mathrm{a}, \mathrm{b}}$, Quy Don Tran ${ }^{\mathrm{a}}$, Nam Nghiep Tran ${ }^{\mathrm{a}, \mathrm{e}^{*}}$, Toan Dinh ${ }^{\mathrm{d}, \mathrm{e}}$, Hoang-Phuong Phan ${ }^{\mathrm{e}}$, Nam Trung \\ Nguyen $^{\mathrm{e}}$, Tran Tung ${ }^{\mathrm{a}}$, Dusan Losic ${ }^{\mathrm{a}}$, Volker Hessel $\mathrm{f}^{\mathrm{a}, \mathrm{f}^{*}}$ \\ ${ }^{a}$ School of Chemical Engineering and Advanced Materials, University of Adelaide, Australia \\ ${ }^{\mathrm{b}}$ Department of Chemical Engineering and Chemistry, Eindhoven University of Technology, the \\ Netherlands \\ ${ }^{\mathrm{e}}$ Department of Chemical Engineering, Can Tho University, Vietnam \\ ${ }^{d}$ Department of Mechanical Engineering, University of Southern Queensland, Australia \\ ${ }^{\mathrm{e}}$ Queensland Micro and Nanotechnology Centre, Griffith University, Australia \\ ${ }_{\mathrm{f}}^{\mathrm{S}}$ School of Engineering, University of Warwick, United Kingdom \\ [*orresponding author]
}

Email: volker.hessel@adelaide.edu.au

namnghiep.tran@adelaide.edu.au

\begin{abstract}
Quantum dots (QDs) were suggested a decade ago as a means of spectral decoy to divert rocket attacks from flying objects such as satellites but never found industrial applications yet. A possible reason is that these advanced nanomaterials are unstable in the long-term under the forces exhibited on a flying object and the nanoparticles might agglomerate to form larger microparticles, which have a different, unwanted spectral footprint. This work proposes an on-board (in-situ) real-time synthesis of quantum dots on a satellite as a result of an interdisciplinary innovation that combines nanomaterial chemistry, heating, and mixing with batch microfluidics, and microfabrication of a miniaturized chip. We employed a microchip as the in-situ reaction chamber due to its capability fast, compact synthesis. The implementation of the micro-reactor, on the other hand, leads to other fundamental challenges that need to be solved as compared to the standard microfluidic chip-based synthesis. Firstly, pumps are far too bulky and heavy for satellite applications. Robust pump-free fluid motion is imperative. Secondly, the synthesis has to be accomplished in less than $180 \mathrm{~s}$, equivalent to the typical time a rocket needs to reach the satellite from the ground. Thirdly, the quantum dots need to be tailored in the type of material and size to replicate the spectral signal of the to-be-protected space asset. Last, the synthesis process needs to be robust and resilient to work reliably and automatically under the demanding conditions of a flying object. As a candidate based on literature proposition In this study, we selected cadmium selenide (CdSe) and lead selenide (PdSe) as the materials of choice. Our synthesis process aims to simplify and tailor CdSe and PdSe quantum dots to match the above demands. With this motivation, the produced quantum dots were characterized by their wavelength emission spectra and high-angle annular dark-field scanning transmission electron microscopy (HAADF STEM) images. While the chosen CdSe-based synthesis was unable to reach IR-emitting sizes within several minutes, we managed to meet all other research targets. Accordingly, $\mathrm{PbSe}$ has been suggested as a new candidate to reach NIR emission within a strict timeframe as reported by Lu et al ${ }^{1}$. Results show that both CdSe and PdSe quantum dots have been successfully synthesized within 10 minutes and provide a spectrum in the UVVisible range of $365 \mathrm{~nm}$ to $850 \mathrm{~nm}$ with an excitation wavelength of $350 \mathrm{~nm}$. This work finally provides insights into the limits of batch synthesis in a microfabricated nanodot chip, such as the need to improve reproducibility and enhance particle growth. Based on this learning, we propose a self-priming flow microchip synthesis can capitalize mixing as a key asset to overcome those limits.
\end{abstract}

Keywords: Quantum dots; Spectral Decoy; Space Assets; Microchip; Synthesis 


\section{Introduction}

Space is becoming increasingly important for humanity. It is thus essential to start considering the vulnerabilities of space assets, which hazards are present, and solutions to mitigate these hazards ${ }^{2-7}$. Threats to space assets, in particular satellites, include those from the natural space environment such as solar storms, while others connect to an artificial source such as cyber-attacks, space debris, directed energy weapons, and physical attacks from extreme countries ${ }^{8-10}$. Currently, no unique countermeasure system is available to protect space from the complete spectrum of these threats. Therefore, innovative and specialized protection means have to be designed.

Physical attacks on satellites manifest as an X-to-space missile where $\mathrm{X}$ indicates surface, air, or space. These anti-satellite weapons have been of great interest for decades. The first successful satellite hit was reported in September 1985 and launched from an F-15 fighter jet ${ }^{11}$. Roughly 20 years later, in January 2007, China deliberately shot down one of its defunct weather satellites. This anti-satellite (ASAT) test received much criticism as the collision created a significant amount of trackable space debris predicted to remain in orbit for decades ${ }^{12,13}$. The question of 'space superiority' was raised once again as the past decade has seen an increase in successful ASAT tests. The USA reportedly shot down one of their defunct reconnaissance satellites in early $2008^{14}$, while Russia took down one of their satellites in November $2015^{15}$, and India showed its ability back in March 2019, claiming that mission Shakti only took 168 seconds $^{16}$.

Designing a proper decoy or protection system relies on understanding the detection mechanisms used by incoming attackers. Various types of precision targeting mechanisms exist such as incorporated satellite navigation, laser guidance, high-definition radars, and advanced radiation-seekers (e.g. infrared $)^{17}$.

Such infrared radiation-seeking missiles, also known as heat-seeking missiles, have been highly effective in target acquisition and annihilation in the past $^{18}$. There are various methods to protect atmospheric assets against these threats, among which a flare is a well-known example. These pyrotechnic compositions are based on magnesium, Teflon, and Viton (MTV-flares) and function as IR-decoy flares ${ }^{19-21}$. However, their capability to generate intense blackbody radiation signatures is inhibited by the anaerobic space environment, rendering combustion-based decoys undesirable. Yet, another suggested protection method is an IR radiation-seeker missile jammer ${ }^{18}$. However, this active defensive system exhibits severe limitations due to the extreme standoff range of $100-1,000 \mathrm{~km}$ and a high target velocity ${ }^{22,23}$. Huang et al. proposed a combination of quick maneuvers with the deployment of IR decoys as a viable defense strategy ${ }^{24}$. Nevertheless, quick and erratic maneuvers are practically difficult in space, especially when the space asset is autonomous.

Considering the daunting challenges in the previous systems, an American defense and technology company, Raytheon, pioneered in employing quantum dots as an optical decoy system. The concept patented by Raytheon overcomes the problems in the conventional systems by the dispersion of a cloud of quantum dots with electro-optical properties ${ }^{25}$. This approach encompasses a passive countermeasure tailored to the to-be-protected space asset that can deceive and dazzle the incoming attacker.

These quantum dots are nanometer-sized particles with geometric nanocrystal radius less than the Bohr exciton radius of the corresponding bulk material, enabling the quantum confinement effect for the exciton ${ }^{26}$. The Bohr exciton radius is the average distance between the electron in the conduction band and the hole in the valence band, where it originated from. The extremely small size of colloidal quantum dots results in the energy levels of the exciton being quantized rather than continuous due to the confinement in three spatial dimensions. The quantum confinement effect allows size-tunable dot emissions ${ }^{26,27}$. Theoretically, a replica of almost any given spectrum can be achieved by a proper combination of quantum dots.

Our present work aims to leverage on the technology previously mentioned in the Raytheon patent and make it more suitable for space applications. The original idea of Raytheon suggests that the 
quantum dots are prefabricated on Earth and subsequently transported into space. However, prefabrication and long-term storage of quantum dots can result in electro-optical decay of the dots, eventually leading to a decrease in the decoy capabilities of the overall system. This is because IRemitting nanodot materials are prone to oxidation from any oxygen remaining after atmospheric production ${ }^{1,28,29}$, which will change the expected decoy emission behavior. Furthermore, the difficulty in stabilizing colloidal quantum dot suspensions can lead to an aggregation of nanocrystals that is problematic for long-term storage ${ }^{26,30,31}$. This issue is especially severe in space as the high-energy and gravity environments, especially during launch, promote aggregation of these colloidal mixtures.

Herein, we propose an in-situ production of quantum dots to avoid the above problems. It is important to define the desired IR-spectrum emitted by the in-situ produced quantum dots. A replica of a satellite spectrum is ideal, yet this is difficult to implement as no clear optical spectrum has been found in the literature. The Raytheon patent provides a satellite spectrum with an onset of roughly $4,000 \mathrm{~nm}$ and a width of over $7,000 \mathrm{~nm}$. The extreme width of this spectrum is impossible to imitate at this point using a single chip. Therefore, we decided to set a new and more specified mock-spectrum goal to steer the chip design. We also approached the MVT-flare, whose peak emission is widely used in aircraft defense mechanism. Thus an emission peak of roughly $1,300 \mathrm{~nm}$ is desirable for our chip ${ }^{32}$.

Moving chemical synthesis from well-known atmospheric conditions to the exo-atmospheric conditions found in space results in several challenges that need to be addressed. Several strict limitations imposed by the predicted synthesis environment thus strongly affect the ultimate reactor design. These challenges include:

- Microgravity: Strongly decreasing gravitational field strength makes non-gravitational effects dominate in handling liquids in space. Effects such as thermosolutal, phase-change, surface/interfacial tension, and electric/magnetic fields dominate over convection ${ }^{33,34}$. The true gravitational acceleration experienced in a spacecraft varies with its location, acceleration, and rotation. Variability is also induced by altitude control maneuvers and vibrations from machinery ${ }^{33}$. This fluctuating microgravity environment severely decreases the reproducibility of chemical synthesis, making a gravity-reliant-batch synthesis undesirable. Furthermore, the strong decrease of natural convection leads to the requirement for complex compensating systems. Active mixing is necessary as a change in density does not induce significant motion as experienced on Earth.

- Temperature \& pressure: The lack of natural convection due to vacuum in space means objects do not cool by thermal convection and conduction. Vacuum hinders the use of outer-space as an external heat-sink. A decoy system will, therefore, experience significant temperature fluctuations. Internal conduction can occur and will decrease the maximum temperature fluctuations. Moreover, low vacuum level space forces many solvents to immediately start boiling as the absolute pressure is below the vapor saturation pressure.

- Weight: Moving chemistry to space drastically increases the overall decoy system operating/launching cost. Recent launch prices (2017) range from roughly 1,500 \$USD per kg for the Falcon Heavy (SpaceX) to 3,000-4,000 \$USD per kg for many of NASA's cargo launchers ${ }^{35}$. Therefore, minimizing the weight of the decoy system is certainly required to suppress the overall cost.

- Limited human interaction: The decoy system is expected to be a part of a small satellite meaning no humans are close-by for maintenance and activation. Furthermore, such a decoy system should be completely autonomous and fool-proof to ensure optimal decoy preparation and timing.

These challenges strongly affect the possibilities of the decoy reactor. We propose to explore a pump-free fluid transport mechanism as a key prerequisite to conducting the synthesis in a small format and in real-time. Leveraging microflow synthesis for this kind of in-situ space manufacturing allows for circumventing the limitations imposed by the microgravitational environment. Surface effects and 
capillary force dominate in microfluidics. Employing microfluidics can significantly reduce overall weight as capillary effects eliminate the need for active and heavy pumps. The lack of gravity is an advantage in these microcapillary systems as the fluid rise is not limited by the gravitational body-force. Moreover, moving to the vacuum of space creates a pressure difference between the enclosed chip chambers and the space environment, allowing for a weightless pumping motion. The temperature and pressure conditions in space limit the decoy system to a stand-alone and autonomous system. Isolating the microchip and removing external influences increases the reproducibility of the quantum dot synthesis, ultimately strengthening the fool-proof nature of the required chip.

Predictability and reproducibility are of key importance as the synthesis needs to perform exactly as expected and designed, even after being standby for a long time. Advances in nanocrystal synthesis have enabled a relatively monodisperse and highly crystalline production process ${ }^{36-38}$. Currently, quantum dots are often prepared using a hot-injection based batch process, in which one precursor is rapidly injected into a heated flask containing the other. The quality of these dots depends on macroscopic effects such as overall temperature and concentration but is also strongly affected by microscopic factors such as concentration fluctuations, local temperature variations, and stirring rates.

A continuous flow system operates in a steady-state regime with enhanced synthesis control and reproducibility. Scaling down the overall reactor dimensions to micrometers increases the surface-tovolume ratio, improving heat and mass transfer, and allows for precise temperature control ${ }^{39,40}$. The benefits of moving to microfluidic synthesis are (I) high surface-to-volume ratio, (II) high heat and mass transfer, (III) efficient mixing, (IV) better temperature control, (V) continuous production, and (VI) a decrease in reagent consumption. Microfluidic devices can significantly reduce the necessary reaction time and thus suit our proposed application. Tian et al. ${ }^{41}$ developed a novel microfluidic method to synthesize full-color emitting CdSe dots and reported a good monodispersity while decreasing the amount of $\mathrm{Cd}$ used. Visaveliya et al. ${ }^{42}$ presented a microfluidic-assisted reproducible synthesis of several fluorescent polymeric nanoparticles with tuned size and surface charge. Moreover, Kubendhiran et $a l .{ }^{43}$ recently published a review about the microfluidic synthesis of semiconducting colloidal quantum dots and their applications.

Moving the entire quantum dot production process to space requires the creation of well-designed syntheses and microchips. From an economic point of view, this approach was considered not feasible at the start of our research. Thus, we focused on proof-of-concept tests with a visible, well-known reaction scheme, rather than a lesser-known but more suitable synthesis. A suitable and relatively simple quantum dot synthesis thus had to be found. Minimizing the number of reaction steps strongly decreases the complexity of the decoy chip. For this, we have chosen to modify the methodology proposed by Kikkeri et $a l^{44}$. This modification entails changing from micro-reactor to batch synthesis, increasing the amount of lauric acid to promote $\mathrm{CdO}$ dissolution, and applying a heating bath for a heat-up batch synthesis.

\section{Experimental section}

\subsection{Materials}

+ CdSe QDs were synthesized using the following chemicals: Cadmium oxide (powder, 99.5\%), Lauric acid (98\%), Oleic acid, Selenium (powder, $\sim 100$ mesh, $>99.5 \%$ trace metal basis), Trioctylphosphine (technical grade, 90\%), Methanol (>99\%), Hexane (anhydrous, 95\%), and Chloroform. All chemicals were ordered from Sigma-Aldrich.

+ PbSe QDs were synthesized using the following chemicals:: Lead (II) acetate trihydrate $(99.999 \%$ trace metals basis), diphenyl ether (ReagentPlus, 99\%), Oleic acid, Selenium (powder, $\sim 100$ mesh, $>99.5 \%$ trace metal basis), Trioctylphosphine (technical grade, 90\%), Methanol (>99\%), Hexane (anhydrous, 95\%), and Chloroform. All chemicals were ordered from Sigma-Aldrich. An argon flow was used to create an inert environment.

\subsection{Methodology}




\subsubsection{Preparation of quantum dots in batch reactor}

Preparation of CdSe QDs: CdSe QDs of different sizes were synthesized according to our slightly modified methodology as proposed by Kikkeri $e t a l^{44}$. The cadmium precursor was prepared by heating cadmium oxide $(100 \mathrm{mg}, 0.75 \mathrm{mmol})$ with lauric acid $(1,000 \mathrm{mg}, 5.0 \mathrm{mmol})$ at $150^{\circ} \mathrm{C}$ until a clear solution was obtained. Oleic acid $(2.0 \mathrm{~mL})$ was added to the solution. After addition, the solution temperature was kept at $150^{\circ} \mathrm{C}$ and stirred at $500 \mathrm{rpm}$ for 10 minutes. The selenium precursor was prepared by adding selenium $(80 \mathrm{mg}, 1.0 \mathrm{mmol})$ to trioctylphosphine $(2.0 \mathrm{~mL})$ and stirred at $500 \mathrm{rpm}$ until the solution turned clear. The synthesis was started by mixing $1.0 \mathrm{~mL}$ of both precursors and heating the mixture to $150^{\circ} \mathrm{C}$. The formation of quantum dots was indicated by a gradual change in color from yellow to deep red. Small samples were taken at a reaction time of 30 seconds, 1, 2, 3, 4, 6, 8 , and 10 minutes.

Preparation of PbSe QDs: PbSe QDs of different sizes were synthesized according to the procedure mentioned by Tan et al. ${ }^{45}$. The lead precursor was prepared by combining diphenyl ether $(5.0 \mathrm{~mL})$, lead acetate trihydrate $(100 \mathrm{mg}, 0.264 \mathrm{mmol})$, and oleic acid $(0.34 \mathrm{~mL}, 1.077 \mathrm{mmol})$. A condenser circulating cold water was applied after which the mixture was stirred at $500 \mathrm{rpm}$ and $100^{\circ} \mathrm{C}$ for 30 minutes. The setup was continuously purged with argon. The selenium precursor was prepared separately by adding selenium $(200 \mathrm{mg}, 2.533 \mathrm{mmol})$ to trioctylphosphine $(1.6 \mathrm{~mL})$ and manually shaken until the selenium was completely dissolved. The selenium precursor was heated to $100^{\circ} \mathrm{C}$ before starting the synthesis. The synthesis was started by adding the selenium precursor to the flask containing the lead precursor. Both the condenser and argon purge were continued throughout the synthesis. The formation of quantum dots was indicated by a gradual change in color from colorless to light brown. Small samples were taken at a reaction time of $1,2,3,4,6$, and 10 minutes.

The PbSe setup employs the same heating method as used for CdSe. The main difference between the two setups is the addition of an argon flow used for PbSe. This as PbSe and its precursors are considered more air-sensitive than CdSe.

2.2.2. Preparation of quantum dots in microreactor

2.2.2.1. Construction of the microreactor

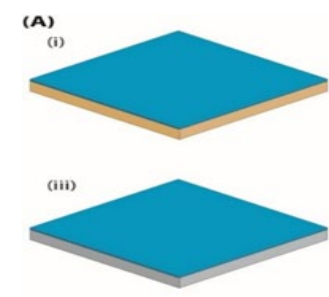

(B)

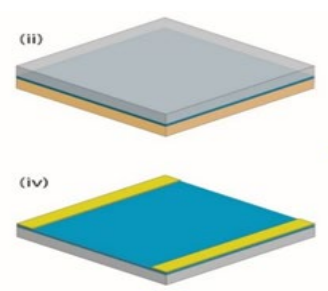

(C)

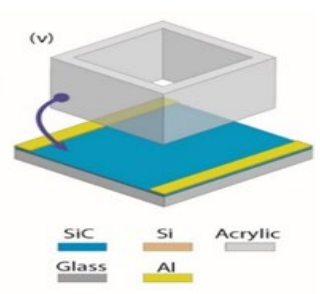

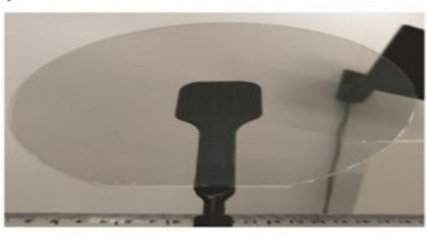

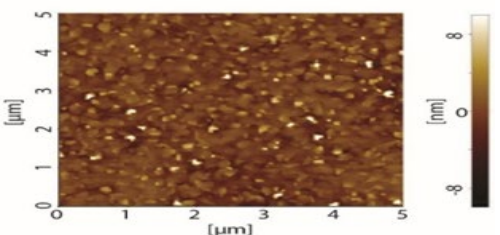

Fig. 1 Fabrication of reaction chamber using silicon carbide. (A) A schematic sketch of the fabrication flow; (B) A photograph of a transparent 6-inch SiC-on-glass wafer; (C) The surface morphology of the SiC film.

We developed the heater for the in-situ synthesis using cubic silicon carbide (3C-SiC) nanothin film, Figure 1A. Silicon carbide was selected due to its chemical inertness that avoids undesirable reactions between the heating element and reactants. Its optical transparency also enables observation of chemical reaction with a standard inverted microscope for earth-based synthesis development. Our fabrication process started with the low-pressure chemical vapor deposition (LPCVD) at $1,250^{\circ} \mathrm{C}$, to epitaxially grow single-crystal $\mathrm{SiC}$ on to standard 6-inch silicon (Si) wafer. The capability of depositing 
thin films of $\mathrm{SiC}$ onto a low cost and ubiquitous $\mathrm{Si}$ substrate can reduce the material cost, while at the same time enabling mass production through wafer-scale-level micromachining. To eliminate the heat conductance to the Si substrate as well as to avoid the current leakage from $\mathrm{SiC}$ to $\mathrm{Si}$ at elevated temperatures, we employed wafer anodic bonding to transfer the $600 \mathrm{~nm}$-thick SiC film onto a glass substrate, Figure 1B. Details for the anodic bonding process and subsequent Si removal can be found in our previous study ${ }^{46}$. The thickness of the film was measured at $600 \mathrm{~nm}$, while its surface morphology was characterized using AFM showing an RMS roughness of below $2 \mathrm{~nm}$, Figure $2 \mathrm{C}$. The carrier concentration was measured using Hall effect measurement and was found to be approximately $10^{19}$ $\mathrm{cm}^{-3}$, sufficiently high to function as a heating element ${ }^{47}$.

We deposited a $300 \mathrm{~nm}$-thick aluminum film onto the $\mathrm{SiC} /$ glass wafer using sputtering. A positive photoresist layer (AZ5214) was then spin-coated and developed, following by Al etching (in $\mathrm{H}_{3} \mathrm{PO}_{4}+\mathrm{HNO}_{3}+\mathrm{CH}_{3} \mathrm{COOH}$ etchant) to form the electrodes. The photoresist was then stripped using $\mathrm{O}_{2}$ plasma and several heaters with dimensions of $10 \mathrm{~mm} \times 10 \mathrm{~mm}$ were diced from the SiC/glass wafer. Subsequently, wire bonding was applied to connect the Al electrode pads to the external copper PCB board for testing. Finally, an acrylic reservoir (patterned using laser machining) with the inner dimension of $6 \mathrm{~mm} \times 6 \mathrm{~mm}$ was attached to the top surface of the SiC chip to constrain the heating chamber.

\subsubsection{Synthesis of QDs in microreactor}

Both CdSe and PbSe microbatch experiments were performed using the synthesis described in the previous two sections. A micro electro-mechanical system (MEMS) microbatch reactor was constructed and used for the synthesis. The total available reaction volume of the microbatch chip is $200 \mu \mathrm{L}$ (see Figure 2). This meant a ratio of 1:1 for the $\mathrm{Cd}$ :Se precursors and 3.35:1 for the $\mathrm{Pb}$ :Se precursor ratio, and a total volume of $100 \mu \mathrm{L}$ for both the $\mathrm{Cd}$ - and Se-precursor in the CdSe microbatch experiment. Volumes of $154 \mu \mathrm{L} \mathrm{Pb}$ - and $46 \mu \mathrm{L}$ Se-precursor were used in the PbSe microbatch synthesis. All precursors were added at their preparation temperature. The CdSe microbatch test was performed for six times: once for HR-STEM analysis, three times at four minutes reaction time, once at six minutes, and once at eight minutes for spectral analysis. The PbSe microbatch was performed four times: once for HR-STEM and three times with a reaction time of one minute for spectral analysis. A batch experiment was done with the remaining PbSe precursors. Preparations for analysis of the quantum dots were performed as described in their respective section. PbSe preparation for HR-STEM followed that as described in the CdSe section.

Heating and power testing: The heating capability of the chip was tested using a DC power supply. The chip was filled with $200 \mu \mathrm{L}$ kerosene (purum). The temperature was measured using a standard hotplate thermocouple, a Minolta/LAND Cyclops mini laser, and a FLIR C2 thermal camera. Two tests were performed to identify the heating behavior. Firstly, the change in temperature was measured at constant wattage. Secondly, a goal of $100^{\circ} \mathrm{C}$ was set and the required heating time was noted at different wattages. The required reaction temperatures could be reached quickly by employing the microheater, making it a viable heating method for microsyntheses.

The microbatch reaction vessel heaters were connected to a DC power supply. The reaction chamber was placed in a plastic box, Figure 2. An active argon flow was applied during the PbSe experiments but this was not done during CdSe experiments. The syntheses were done at atmospheric pressure. 


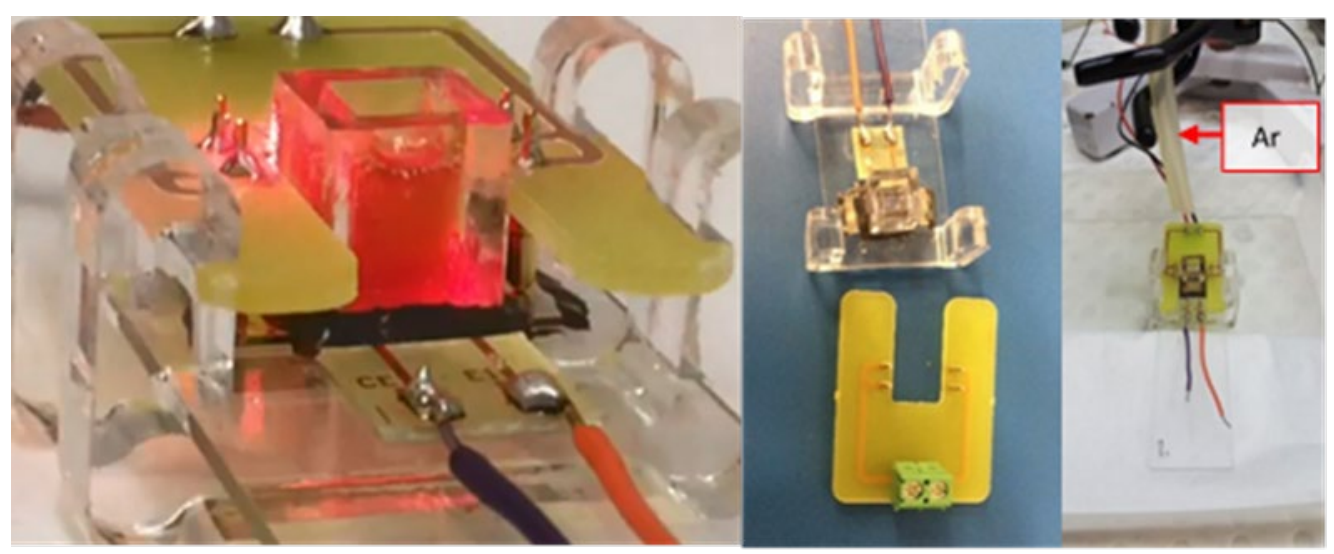

Fig. $2 C d S e$ \& PbSe microbatch setup. (a) IR temperature measurement, with a red laser light illuminating the reaction chamber. (b) an inert atmosphere was created by an active argon flow

\subsection{Sample analysis}

Analysis of optical properties: The CdSe fluorescence emission spectrum was measured by a Horiba FluoroMax-4 Spectrofluorometer. No alterations have been done to the chemical constituents except dilution necessary for proper analysis. All measurements were performed with the corresponding CdSe quantum dots diluted and dispersed in chloroform.

The PbSe NIR fluorescence spectra were acquired using an FLS980 Photoluminescence Spectrometer. A Fianium "Whitelase" MHz picosecond pulsed source with a range from 420-2,400 nm was used in combination with a Hamamatsu R5509-72 photomultiplier (600-1,700 nm detection). The visible PbSe fluorescence spectra were acquired using a Horiba FluoroMax-4 Spectrofluorometer. No alterations have been done to the chemical constituents except dilution in chloroform. The visible absorption spectrum was obtained using a Cary $60 \mathrm{UV}-\mathrm{Vis}$ Spectrophotometer. The same chloroform dilutions were used for visible absorbance and fluorescence spectroscopy.

Analysis of structural properties: HAADF STEM images were acquired using an aberrationcorrected FEI Titan Themis $80-200$ operating at $200 \mathrm{kV}$, with a beam convergence semi angle of 25 mrad and HAADF collection angle from 56-200 mrad. Elemental maps were acquired using a superX detector and a low-background sample holder. The quantum dots were purified for this analysis to avoid microscope contamination. The quantum dots of interest were quenched in $1 \mathrm{~mL}$ methanol. The new solution was centrifuged at $5,000 \mathrm{rpm}$ for 5 minutes. The supernatant was removed by pipette and the precipitate was redispersed in $1 \mathrm{~mL}$ methanol. The new solution was sonicated for 40 seconds after which it was centrifuged at $5,000 \mathrm{rpm}$ for 5 minutes to precipitate the quantum dots. The supernatant was removed and the precipitate was redispersed in $1 \mathrm{~mL}$ hexane then to be sonicated for a further 40 seconds.

\section{Results and Discussion 3.1. CdSe quantum dots}

The CdSe quantum dots were produced as outlined in the experimental section. Analysis of their key properties was done within a short time after production to avoid any potential degradation. The final product was analyzed as-synthesized; thus no purification steps were performed unless necessary. We decided to use the unpurified product as this would most closely simulate the real conditions in which the quantum dots would perform. The quantum dots dispersed into space to mimic a space asset radiation signature would not be purified due to the stringent time limit, yet it is to be expected that the solvents will boil-off in the vacuum of space. Hence, it is crucial to analyze the samples as-synthesized and consider the effects of each solvent.

The samples in this study were synthesized with different reaction times. A limit of 10 minutes reaction time for the batch reaction was imposed because of the strict time limit imposed by mission 
Shakti, which is 168 seconds. Figure $3 \mathrm{~b}$ shows a visual validation proving the creation of visible-light emitting quantum dots. Figures $3 \mathrm{a}$ and $3 \mathrm{~b}$ indicate that the methodology presented in the previous section has successfully synthesized CdSe quantum dots of different sizes and emissions due to a change in reaction time. The results show that our reaction scheme promotes quick nanoparticle nucleation yet takes significant time to start noticeable particle growth, as indicated by the high number of blueemitting samples. It must be noted that the apparent change in color from samples 2.0 to 3.0 minutes, as shown in Figure 1b, is due to the reflection of light emitted by the 4.0-minute sample. Actually, high reaction temperatures $\left(\sim 300^{\circ} \mathrm{C}\right)$ usually result in fast nucleation, and large nanocrystals are quickly obtained. In this study, at low temperatures which is $150^{\circ} \mathrm{C}$, the size of the nanocrystals and the concentration of the unreacted precursors in the mixture can be balanced. Thus, continuous nucleation is suppressed, the residence time distribution (RTD) is narrowed, and homogeneous QD fractions are obtained by varying the reaction time. The homogenous reaction mixture and slow nucleation results in a mild process for the production of QDs using microreactors.

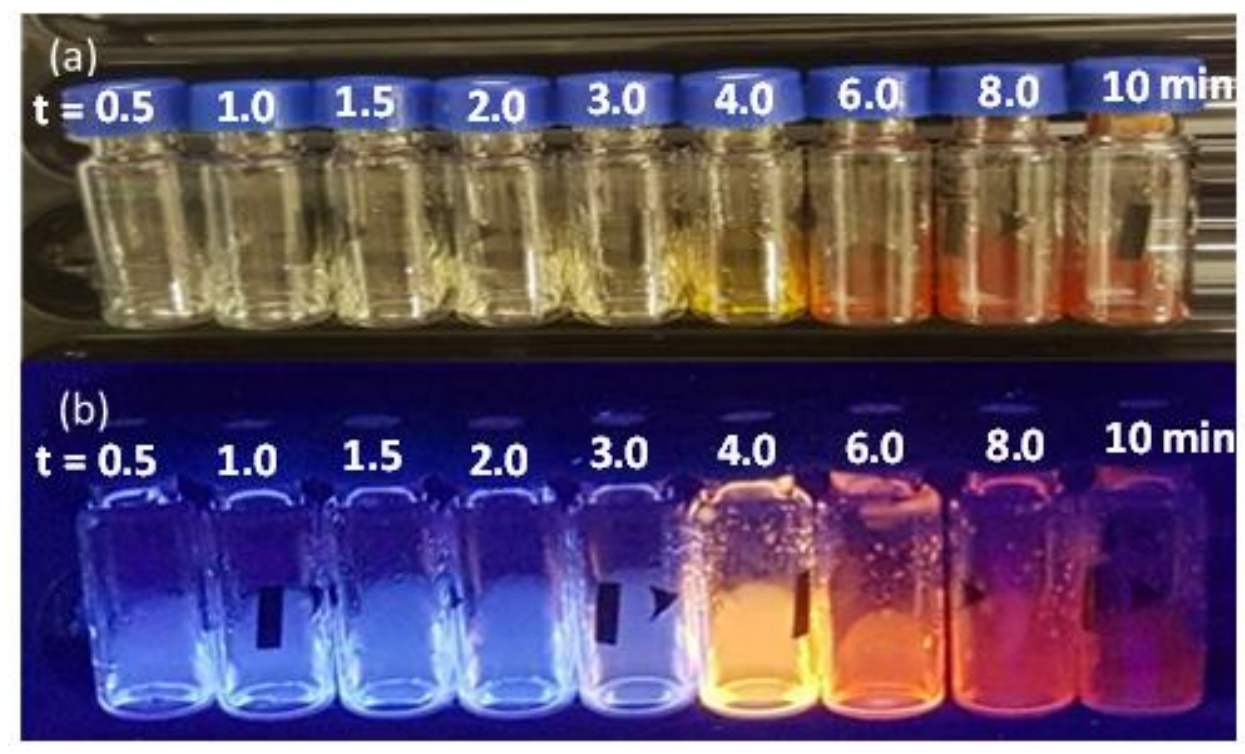

Fig. 3 Synthesized CdSe quantum dots in (a) room light and (b) excited at $350 \mathrm{~nm}$. An increase in wavelength is shown for increasing reaction times.

One can explain the time difference between nucleation and growth by noticing that the chosen reaction scheme is considered a colloidal heat-up synthesis route. The classical colloidal route consists of three major processes: (I) monomer generation resulting in (II) nucleation followed by the (III) growth of seeds ${ }^{26,36}$. The chosen heat-up approach is a single-pot nanocrystal synthesis where all constituents are mixed before heating. Heating the mixed solution initiates both nucleation and successive particle growth. Thermal degradation of the precursor into monomer increases the concentration, eventually reaching the critical nucleation concentration. There is an energetic barrier present that needs to be overcome to start seed nucleation. This separates nucleation from growth as the monomer concentration drops after the nucleation event. The immediate nucleation followed by slow growth of our samples indicates that this nucleation threshold was reached early due to the separate precursor preparation. The subsequent reaction temperature was not high enough to sufficiently promote new monomer generation and particle growth. Therefore, preheating the precursors is suggested for the next quantum dot material.

\subsubsection{CdSe optical properties}

The samples were analyzed for their emission spectrum in the UV-Visible range of $365 \mathrm{~nm}$ to $850 \mathrm{~nm}$ with an excitation wavelength of $350 \mathrm{~nm}$, shown in Figure 4. Furthermore, the peaks and FWHM observed for each sample are tabulated in Table 1. The results show that the observed wavelength emission peak of each sample corresponds to the luminescent property observed under the 
UV-light. The analyzed peaks are broad compared to those reported by Kikkeri et al ${ }^{44}$, possibly due to the simplification of our reaction scheme. Our CdSe quantum dots are highly tunable for emission yet are unable to reach the predetermined emission goal within the set timeframe of 10 minutes. The samples with a reaction time of 6,8 , and 10 minutes appear to start forming a bimodal distribution as indicated by the change in slope. This apparent bimodal distribution is caused by the increasing inaccuracy of the FluoroMax-4 at higher wavelengths, leading to a strong increase in the emission correction factor of the instrument.

Figure 4 also shows a delayed growth time followed by a quick but decreasing growth rate. The growth rate for nanocrystal dots depends on many parameters, including their size. The temporal evolution of the size-dependent growth rate of nanocrystalline dots is negative ${ }^{48}$. That means the smaller particles grow quicker than larger particles. Defining the particle growth rate as the radius increment per unit time suggests that a particle growth-rate curve indeed has a negative slope and the standard growth speed decreases over time ${ }^{36}$. We would expect the standard deviation of the size distribution, also known as the FWHM, to decrease too but the opposite appears to be the case. This result was also noted by Kikkeri et $a l^{44}$. The solvents were not investigated because the chosen CdSe-based synthesis was unable to reach IR-emitting sizes within several minutes while meeting the other research targets. Such investigation into solvent behavior should include both fluorescence and absorbance spectroscopy to show whether the chosen solvents are optically suitable for our protection system.

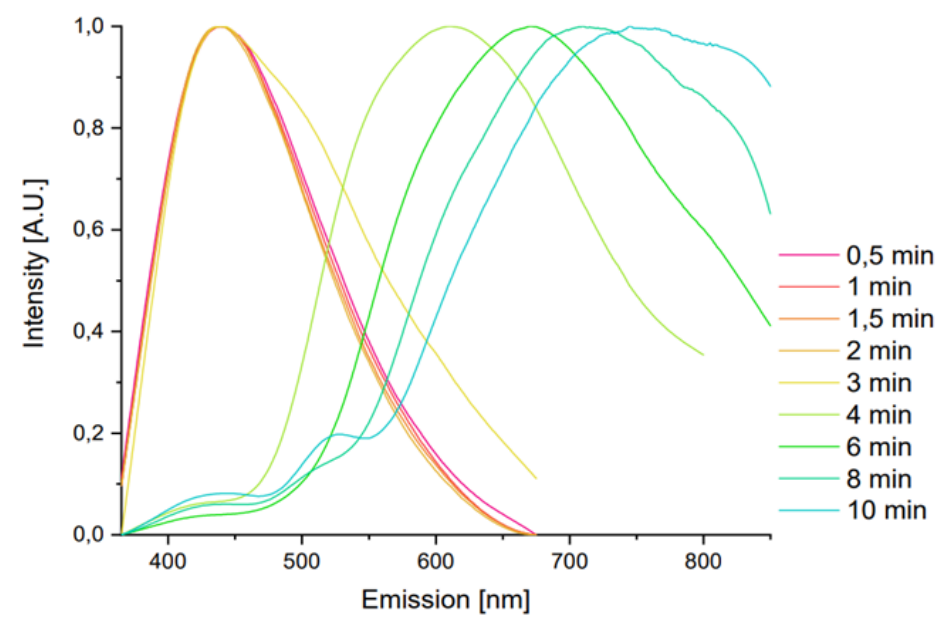

Fig. 4 CdSe fluorescence spectra excited at $350 \mathrm{~nm}$. Graph is normalized for each peak. CdSe shows strong size-tunable emission as expected

Table 1 Fluorescence wavelengths of different reaction times and corresponding FWHM

\begin{tabular}{lcc}
\hline Sample & $\begin{array}{c}\text { Emission Wavelength } \\
\text { Peak (nm) }\end{array}$ & $\begin{array}{c}\text { FWHM } \\
\text { (nm) }\end{array}$ \\
\hline $0.5 \mathrm{~min}$ & 442 & 144 \\
$1 \mathrm{~min}$ & 442 & 141 \\
$1.5 \mathrm{~min}$ & 443 & 137 \\
$2 \mathrm{~min}$ & 443 & 137 \\
$3 \mathrm{~min}$ & 445 & 174 \\
$4 \mathrm{~min}$ & 610 & 228 \\
$6 \mathrm{~min}$ & 671 & 270 \\
$8 \mathrm{~min}$ & 718 & -- \\
$10 \mathrm{~min}$ & 749 & -- \\
\hline
\end{tabular}




\subsubsection{CdSe degradation}

The emission spectra of the 4- and 8-minute samples were analyzed at the time of synthesis and after 9 days of storage. Both samples were stored in identical glass containers under identical laboratory conditions. Such a test was performed to gain a deeper understanding of the degradation associated with storage time and to indicate whether the in-situ and real-time production of quantum dots is indeed necessary. Figure 5 shows the emission spectra of the samples. The corresponding peak values are tabulated in Table 2. A blue-shift in the emission peak of $16 \mathrm{~nm}$ from $610 \mathrm{~nm}$ to $594 \mathrm{~nm}$ was observed for the 4-minute sample and a shift of $103 \mathrm{~nm}$ from 718 to $615 \mathrm{~nm}$ for the 8-minute sample. This blueshift indicates a change in particle size. We conclude that a dispersion merely based on the steric hindrance provided by oleic acid and trioctylphosphine as surface ligands do not provide sufficient stability for long-term storage as expected. Even though it is not possible to determine an exact decay speed, Figure 5 does show the need for real-time quantum dot production.

A high-resolution STEM analysis has been performed on these samples. The quantum dots were purified as described in the experimental section. The sonication used for purification might change the original morphology, and we, therefore, assumed that the STEM pictures are a 'best-case scenario', being as close to the synthesis result as can be.

The STEM and HAADF images show the coagulation of quantum dots for both samples. An increasing amount of interparticular material bridges was formed. Figure 6 shows the STEM images of a 4- and 8-minute sample after 9-day storage, and a fresh sample from a different batch synthesis with a reaction time of 4 minutes. Figure 7 shows HAADF images obtained from the 9-day storage of a 4minute sample. The HAADF elemental analysis shows that the interparticular bridges are indeed CdSe. The bridge formation can thus explain the apparent emission blue-shift: CdSe material is moved from particles to connections, decreasing the particle sizes. This movement of material has been seen and used in more controlled environments e.g. for the asymmetric Ostwald ripening of quantum dots to $\operatorname{rods}^{49,50}$. Correspondingly, the formation of interparticular CdSe bridges leads to a decrease in FWHM, as tabulated in Table 2, insinuating that bigger particles deposit more strongly towards bridge formation than smaller particles.

Figure 8 shows a comparison of the particle size distribution of the fresh and 9-day old 4-minute sample. The change in particle size distribution agrees with the other results. The most obvious changes appear around the extremes of the distribution. A decrease of the largest particle sizes is seen as well as a strong increase in particle sizes around $2 \mathrm{~nm}$. Though, the difference in the number of particles counted must be noted; $n=939$ for 'Fresh' and $n=209$ for '9-day Old'. This difference explains the apparent roughness of the 9-day distribution as compared to the fresh distribution. The 'Fresh' distribution has a mean of $3.16 \mathrm{~nm}$ with a standard deviation of $0.72 \mathrm{~nm}$, whereas the '9-day Old' distribution showed a decrease in the average particle size of $10 \%$. A mean of $2.82 \mathrm{~nm}$ and a standard deviation of $0.62 \mathrm{~nm}$ was found after storage. These values agree with the general decrease in both particle size and FWHM as seen by optical analysis.

Table 2 Fluorescence wavelengths of different reaction samples and corresponding FWHM

\begin{tabular}{ccc}
\hline Sample & Emission Wavelength & FWHM \\
& Peak (nm) & $(\mathbf{n m})$ \\
\hline
\end{tabular}




\begin{tabular}{ccc}
\hline 4 min fresh & 610 & 228 \\
4 min 9-day old & 594 & 200 \\
8 min fresh & 718 & -- \\
8 min 9-day old & 615 & 242 \\
\hline
\end{tabular}

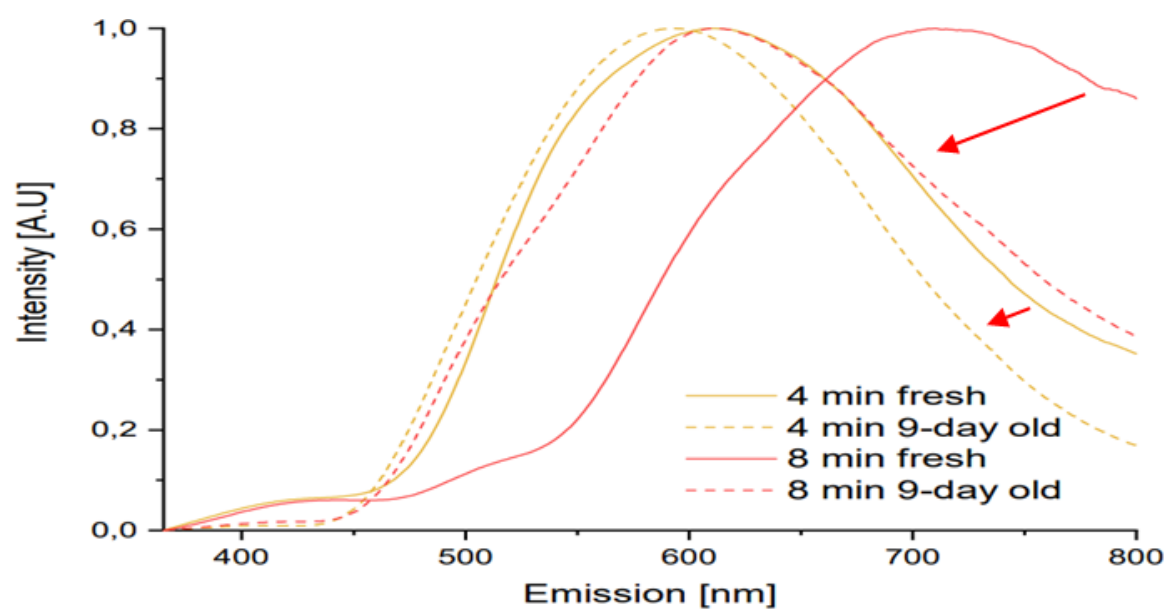

Fig. 5 Fluorescence spectra of fresh and aged CdSe excited at $350 \mathrm{~nm}$. The graph is normalized for each peak. A notable emission change is visualized.

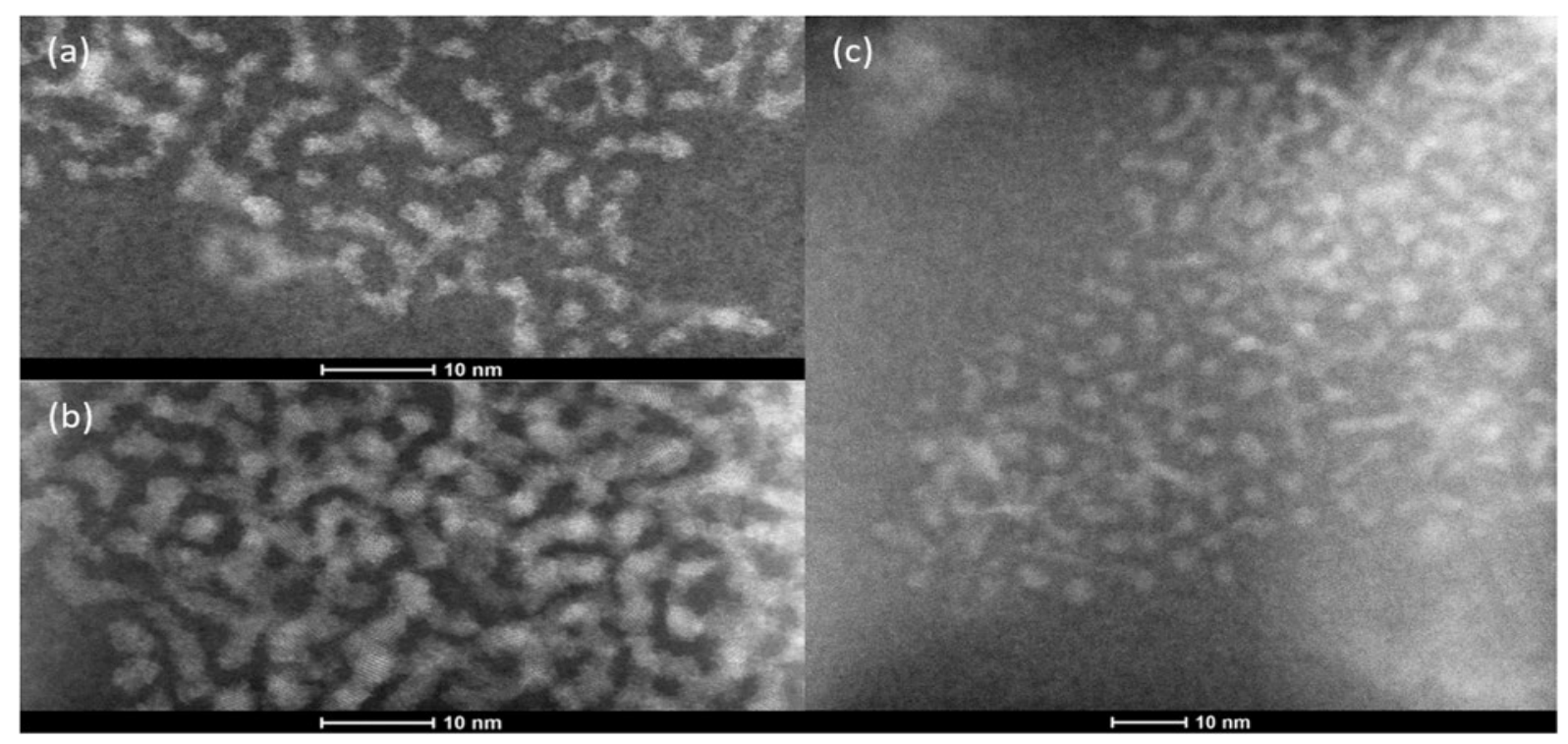

Fig. 6 STEM images of (a) 9-day old sample with a reaction time of 4 minutes, (b) 9-day old sample with a reaction time of 8 minutes, and (c) a fesh sample with a reaction time of 4 minutes. A strong coagulation of particles is shown. 


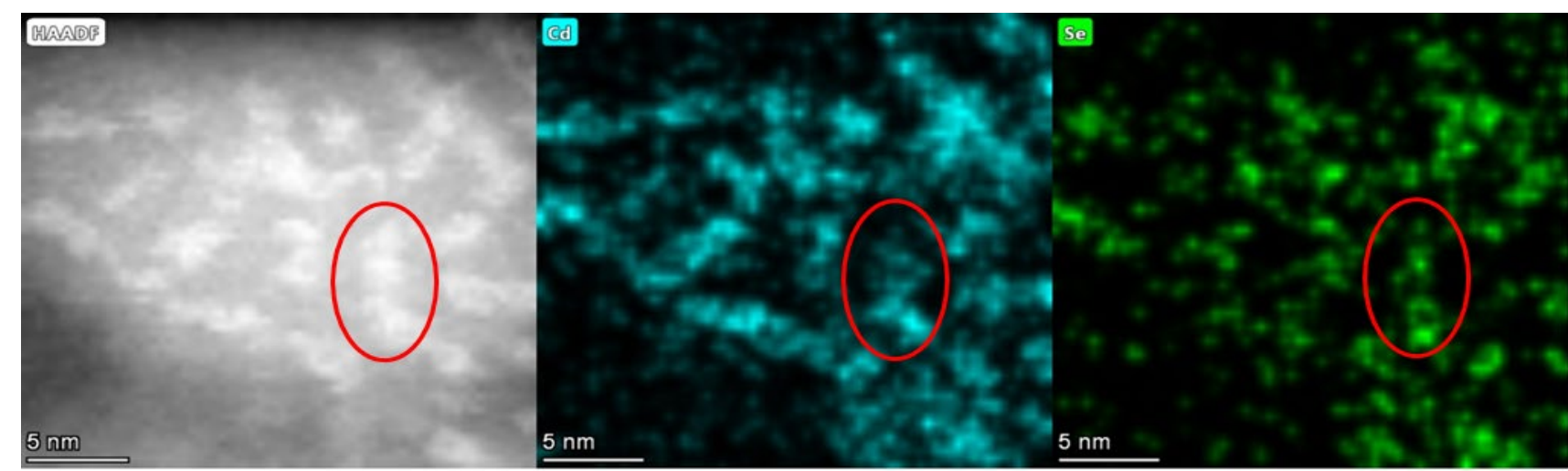

Fig. 7 HAADF and elemental analysis of the 9-day old sample with a reaction time of 4 minutes showing that both $\mathrm{Cd}$ and Se are present in the bridges. The red circles exemplify a bridge

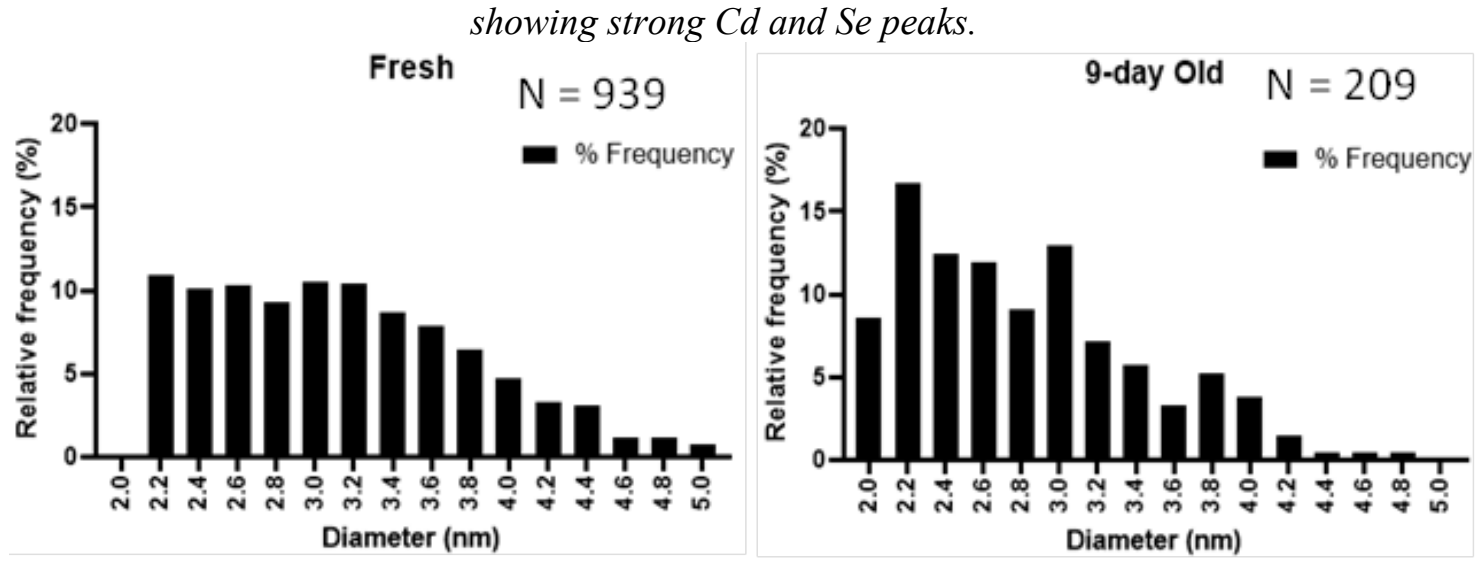

Fig. 8 Particle size distribution of (a) 4 min fresh sample with 939 measured dots having $\bar{x}=3.156$ $\mathrm{nm}, \sigma=0.716 \mathrm{~nm}$ (b) 4 min 9-day old sample with 209 measured dots having $\bar{x}=2.817 \mathrm{~nm}$, $\sigma=0.618 \mathrm{~nm}$. A decrease in average particle size is noted.

\subsection{PbSe quantum dots}

The PbSe quantum dots were produced as outlined in the experimental section. The synthesis chosen was a hot-injection method with both precursors preheated to the corresponding temperature. This to avoid the previously mentioned nucleation/growth time gap. Analysis of their key properties was done within a short time after production to avoid any potential quantum dot degradation. The final product was analyzed as-synthesized, thus no purification steps were performed unless necessary. We decided to use the unpurified product as this would most closely simulate the real conditions, where the quantum dots would perform as a decoy without further purification. The quantum dots dispersed into space to mimic a space asset radiation signature cannot be purified due to the stringent time limit, yet it is to be expected that the solvents will boil-off in the vacuum of space. Hence, it is crucial to analyze the samples as-synthesized and consider the effects of each solvent.

The samples in this study were synthesized with different reaction times. A limit of 10 minutes reaction time for the batch reaction was imposed because of the strict time limit imposed by mission Shakti. In contrast to the CdSe quantum dots, a quick visual check was not possible because PbSe emits in the invisible NIR region. Though, the color change of the reaction mixture indicated a successful quantum dot synthesis.

\subsubsection{PbSe optical properties}

An excitation vs emission plot was acquired to gain an overview of the optical PbSe behavior. For this, a 1-minute reaction time sample was analyzed by being excited from $450-800 \mathrm{~nm}$ while 
measuring the fluorescent emission ranging from 1,000 - 1,500 nm. Figures $9 \mathrm{a}$ and $9 \mathrm{~b}$ show the normalized 3D curve and flat surface, respectively. A peak emission intensity was determined at 1,160 $\mathrm{nm}$ with peak excitation intensity at $530 \mathrm{~nm}$. Therefore, an excitation wavelength of $530 \mathrm{~nm}$ was used for the acquisition of other fluorescence spectra. $530 \mathrm{~nm}$ is a useful wavelength as the emission spectrum from our sun peaks in intensity around $500 \mathrm{~nm}$ (Wien's law). The second peak with an emission peak of 1,120 nm appears to indicate a bimodal size distribution. This is, however, not the case as further experiments did not show this peak. It was concluded that this second peak was created due to a mishap in the synthesis initiation, more specifically the addition of the selenium precursor where it was added in two steps due to failure in using the syringe. Furthermore, it is shown that $\mathrm{PbSe}$ is excited by wavelengths over $800 \mathrm{~nm}$, yet it was deemed unnecessary to test this considering the intensity measured at $800 \mathrm{~nm}$ was well below $10 \%$ of the maximum intensity already.

A new batch of samples was analyzed for their fluorescence emission spectrum in the NIR range of $700 \mathrm{~nm}$ to $1,700 \mathrm{~nm}$ as a result of particle excitation at $530 \mathrm{~nm}$. The samples were used as retrieved from the synthesis, no purification was done. In addition to the spectra in Figure 10, the peaks and FWHM observed for each sample are tabulated in Table 3. The relatively low reaction temperature of $100^{\circ} \mathrm{C}$ was effective and preheating the precursors indeed removed the nucleation/growth time gap present in the heat-up CdSe batch synthesis. The PbSe synthesis, therefore, deems suitable to reach our mock-spectrum. Figure 10 shows, in agreement with that reported for CdSe (Figure 4), a decreasing growth rate. Furthermore, the FWHM ultimately also broadens for PbSe particles.
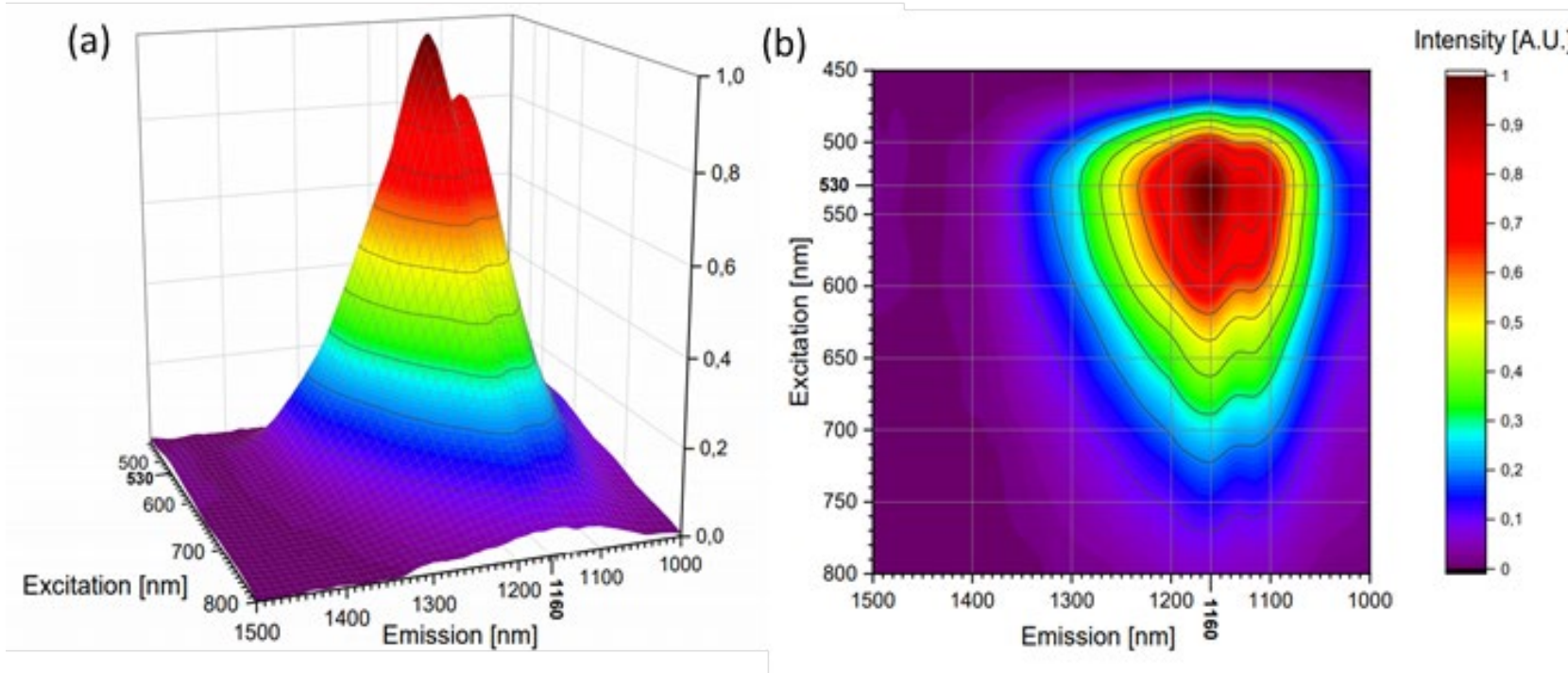

Fig. 9 PbSe excitation vs emission (a) $3 D$ and (b) surface plot. The sample reaction time was 1 minute. Graphs are normalized to maximum PbSe fluorescence intensity. 


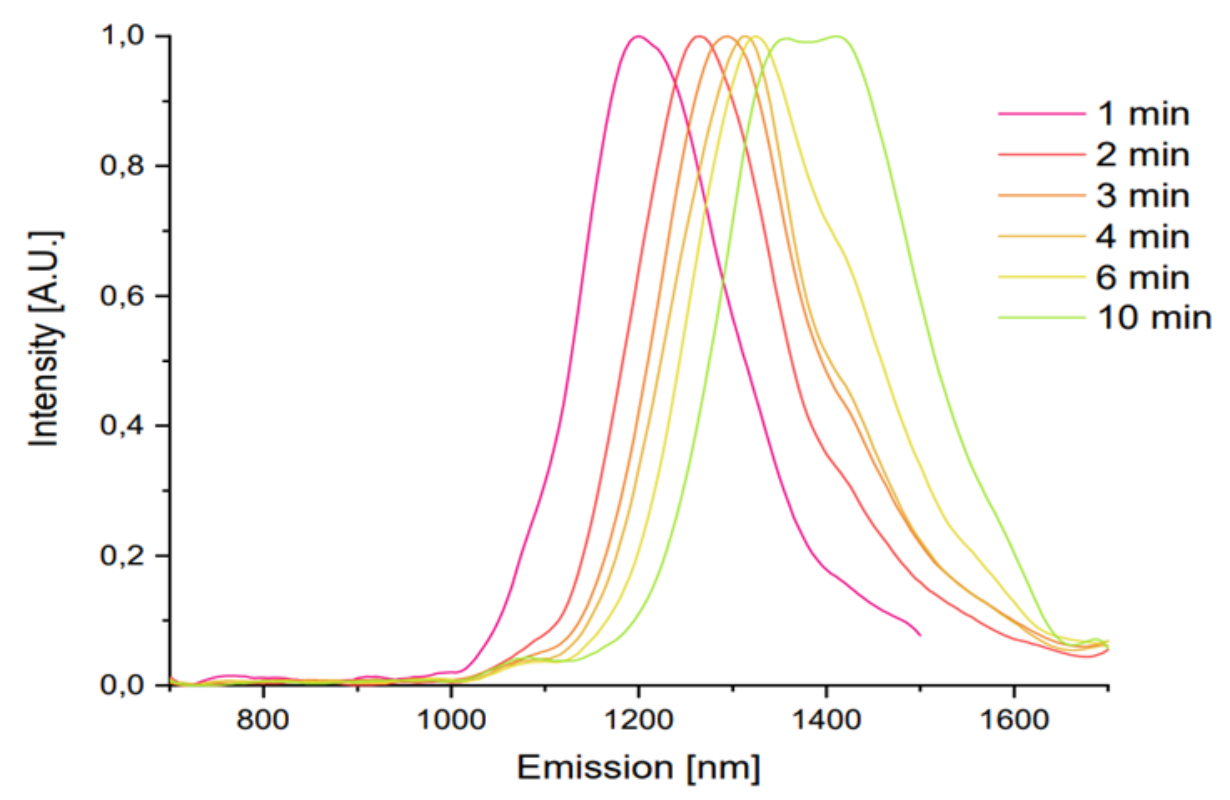

Fig. 10 PbSe fluorescence spectra excited at $530 \mathrm{~nm}$. Graph is normalized for all peaks. PbSe shows strong size-tunable emission as expected

Table 3 Fluorescence wavelengths of different reaction times and corresponding FWHM

\begin{tabular}{lll}
\hline Sample & $\begin{array}{l}\text { Emission Wavelength } \\
\text { Peak (nm) }\end{array}$ & $\begin{array}{l}\text { FWHM } \\
\text { (nm) }\end{array}$ \\
\hline 1 min & 1200 & 180 \\
2 min & 1365 & 175 \\
3 min & 1295 & 185 \\
4 min & 1315 & 180 \\
6 min & 1325 & 215 \\
10 min & 1410 & 240 \\
\hline
\end{tabular}

\subsubsection{PbSe solvent properties}

Further investigation into the solvent behavior was done to check for visible and NIR fluorescence and absorbance. This to show whether the chosen solvents are optically suitable for our protection system: extra fluorescence peaks are unwanted, so is visible absorption as this will decrease the amount of light available for PbSe particles. The solvents tested were oleic acid (OA), diphenyl ether (DPE), and trioctylphosphine (TOP), all of which found use in the PbSe synthesis. Figure 11 shows visible light absorbance spectra, which are normalized to the maximum absorbance of the sample. The spectra show a strong UV-absorbance due to oleic acid, but no significant absorbance due to diphenyl ether nor trioctylphosphine. The absorption percentage in the mixture due to oleic acid increases when nearing the UV-region, indicating a decrease in PbSe excitation activity in the deep blue region. Removing these values from the PbSe line by a simple subtraction shows that PbSe particles indeed show a decrease in UV-excitation activity. Moreover, it is shown that PbSe does absorb visible light, as was previously indicated by the emission vs excitation graph (Figure 9). It is worth noting that these graphs do not agree with each other. Figure 7 shows a maximum excitation response at $530 \mathrm{~nm}$, whereas Figure 11 shows a maximum absorbance at $435 \mathrm{~nm}$. This disagreement is due to the simple and incorrect subtraction performed to obtain Figure 11. The subtraction does not take the actual solvent 
ratio into account as an exact ratio is unknown and this subtraction is, strictly speaking, incorrect. The subtraction merely provides an indication that $\mathrm{PbSe}$ absorbs visible light yet is not the only constituent absorbing light. Figure 9 shows the actual excitation vs emission behavior.

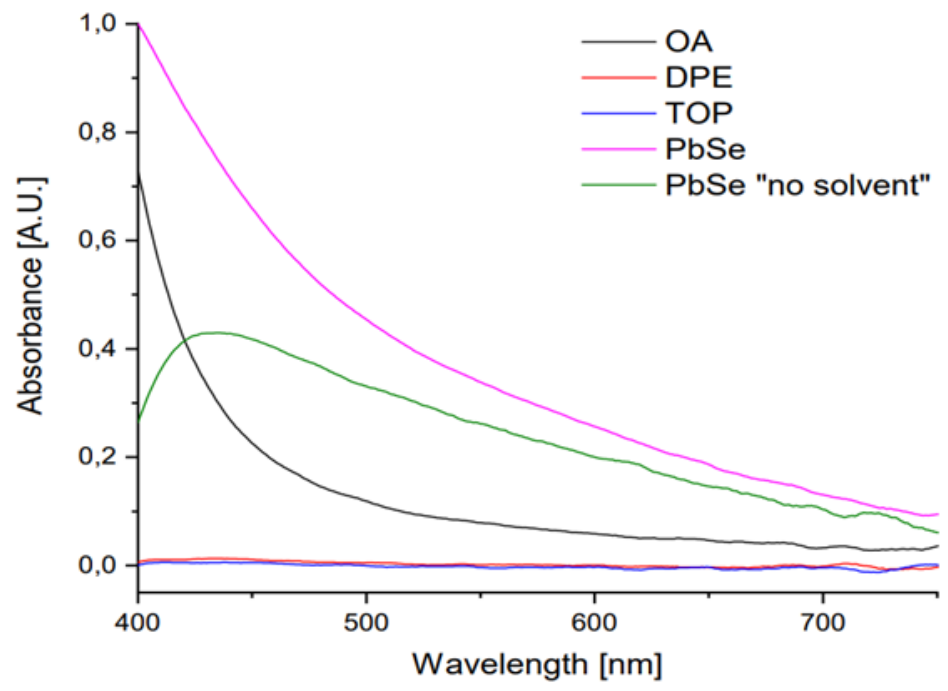

Fig. 11 Visible light absorbance of PbSe and solvents. Graphs is normalized to maximum value of PbSe. Significant UV-absorbance is shown for oleic acid

Figure 12 shows the IR-fluorescence spectra obtained for the solvents excited at $530 \mathrm{~nm}$ to check whether the emitted IR spectrum is completely caused by PbSe particles or the solvents. The emission of diphenyl ether, oleic acid, and trioctylphosphine was measured from $700 \mathrm{~nm}$ to $1,700 \mathrm{~nm}$ and normalized to the average peak value of the PbSe dots. Chloroform (Chloro) was also checked because it serves as a cleaning agent in-between tests. Testing chloroform mitigates any emission resulting from the remaining cleaning agent. Moreover, chloroform is known to be a strong absorber in the NIR-spectrum yet showed no significant fluorescence activity in this region. None of the solvents exhibited a strong NIR fluorescence. Chloroform was not tested in the visible absorbance spectrum as it was used to dilute all samples. The change in fluorescence intensity from 1,380 nm onwards is due to a grid change within the machine.

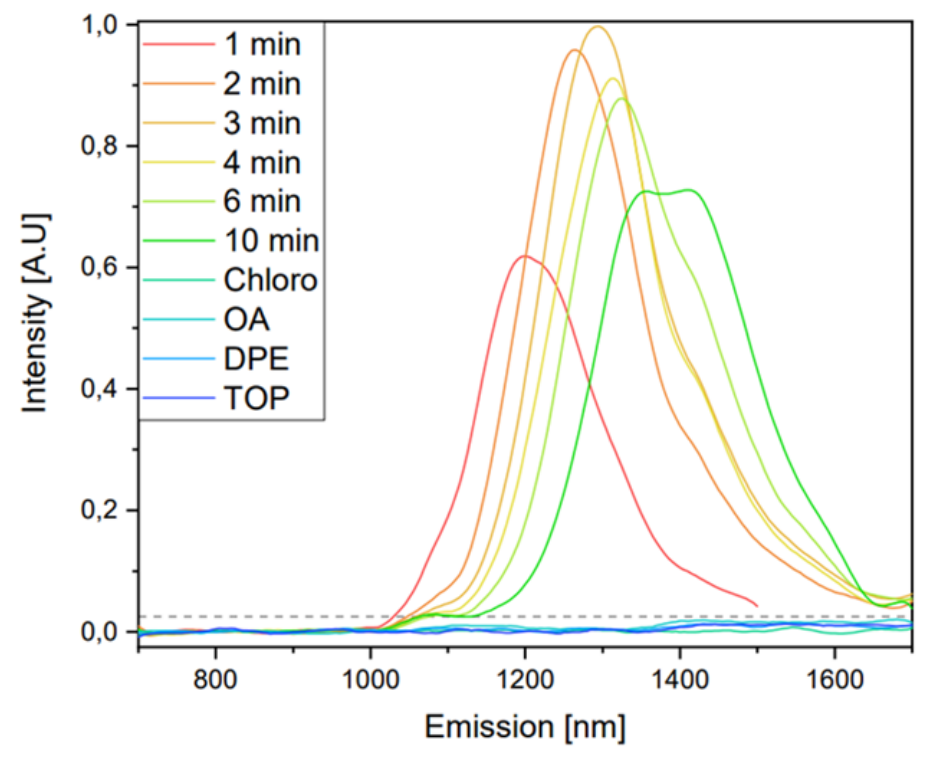

Fig. 12 NIR fluorescence of PbSe synthesis solvents and chloroform. Values are normalized to the average PbSe peak fluorescence intensity. None of the used solvents exhibit notable IRfluorescent emission. 
Lastly, the visible fluorescence of all solvents and one PbSe sample excited at $530 \mathrm{~nm}$ was measured from $365 \mathrm{~nm}$ to $700 \mathrm{~nm}$ to check whether an incoming missile would measure a pure NIR spectrum or a dual-peak spectrum with VIS/NIR emissions. Figure 13 shows the visible fluorescence spectrum with peak emission at $580 \mathrm{~nm}$ resulting from oleic acid excited at $530 \mathrm{~nm}$. Oleic acid emission intensity is negligible compared to the CdSe emission, yet is significant compared to other PbSe related solvents. Figure 13 is normalized to the average value of the CdSe ( $350 \mathrm{~nm}$ excitation) to relate the solvent fluorescence values to a known emission intensity. This peak should pose no problem considering the $\mathrm{PbSe}$ particles are strongly excited by wavelengths ranging from $500 \mathrm{~nm}$ to $600 \mathrm{~nm}$ as shown in Figure 7. The oleic acid emission is mostly absorbed by the PbSe particles as shown by the 1minute PbSe sample in Figure 13.

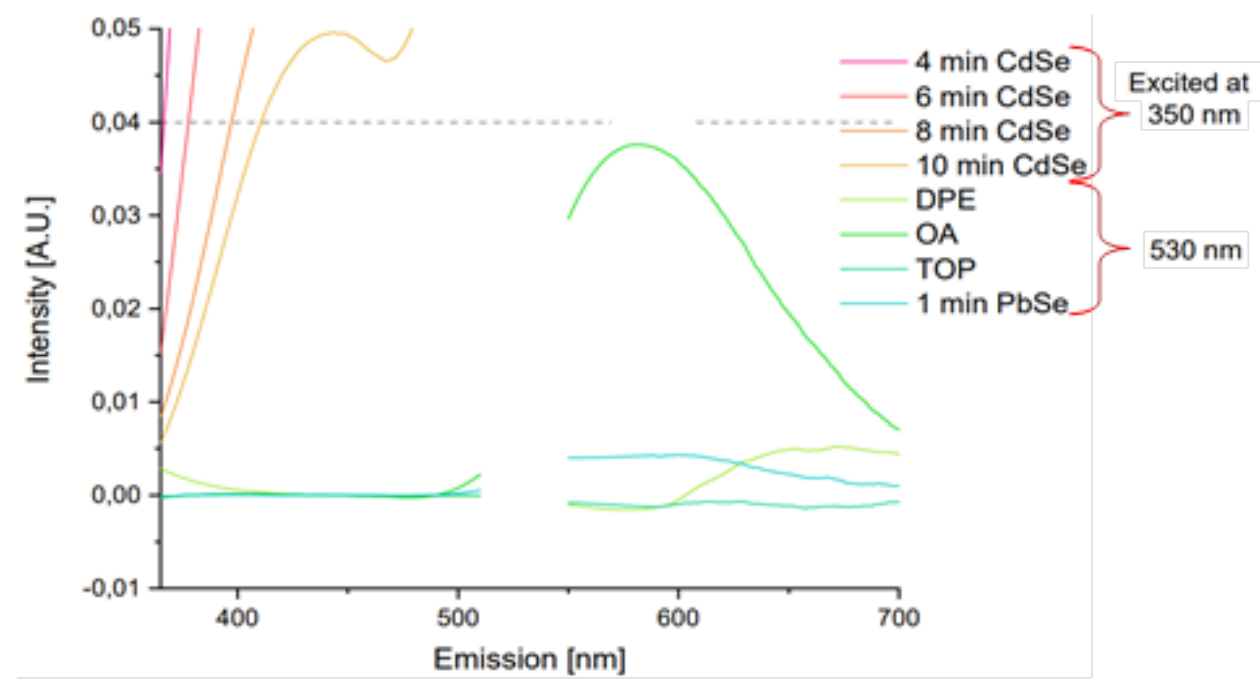

Fig. 13 Visible fluorescence of PbSe and solvents. Values are normalized to the average CdSe peak fluorescence intensity. The visible fluorescent emission is negligible considering the excitation wavelength of $530 \mathrm{~nm}$.

\subsubsection{PbSe synthesis in a microbatch chip}

Having developed the reaction scheme, delivering nanodots of the right spectral footprint for the application considered here, the next step is developing a microfluidic synthesis on a satellite under the outer space conditions. The scope of this paper cannot cover all complex space challenges for a microfluidic synthesis; such as the automatic self-priming, mixing and synthesis, which are left for future works. What can be delivered as the first step is developing and validating a heat-up procedure in a compact-format microchip facing the harsh, space-cold conditions and the stringent time requirement to react to a missile attack. In this sense, and to simplify the reaction scheme to pragmatic process design for a microchip experiment, we added both precursors at the same time in a microbatch with an integrated heater and then heated them together to reach the necessary reaction temperature.

We started with a well-established nanodot synthesis, which is the formation of CdSe, since this allows us to make a straight-forward validation if a synthesis in a microbatch chip with an integrated heater can reveal the same material quality as a well-established batch synthesis. The CdSe was performed six times: once for HR-STEM analysis, three times with a reaction time of four minutes, and at six and eight minutes for spectral analysis. Three experiments with an equal reaction time will give a decent indication of microchip synthesis repeatability. A reaction time of four minutes was chosen as this is the time at which the first particle growth was acquired in the normal batch synthesis.

Therefore, we expected that the spectra would be similar to those from an ordinary batch experiment (peak emission at $610 \mathrm{~nm}$ ). However, a visible inspection of the particles showed a blue color rather than the expected yellow/orange, only the 8-minute sample had a very slight change in color, Figure 14. More detailed fluorescence analysis showed a bimodal distribution for all CdSe 
microbatch experiments. All 4- and 6-minute samples show a respective emission peak around both 443 $\mathrm{nm}$ and $505 \mathrm{~nm}$, Figure 14. A reaction time of eight minutes showed slight particle growth; the main emission peak shifted towards $537 \mathrm{~nm}$.

Thereafter, the CdSe microbatch experiments show excellent repeatability yet no significant particle growth until extended reaction times, yet the particle growth is slower than that of a normal batch synthesis. Also, the sample emissions appeared to be much dimmer than those of normal batch synthesis. The mentioned bimodal distribution agreed with the result from preparing the first microbatch sample for HR-STEM analysis: the quantum dot mixture changed from blue to green emission after centrifuging, Figure 15. This indicates that both colors were present in the original mixture, yet the heavier green particles were more strongly affected by the centrifugal force. HR-STEM analysis was not performed on this sample as the sample changed too much from the preparation.

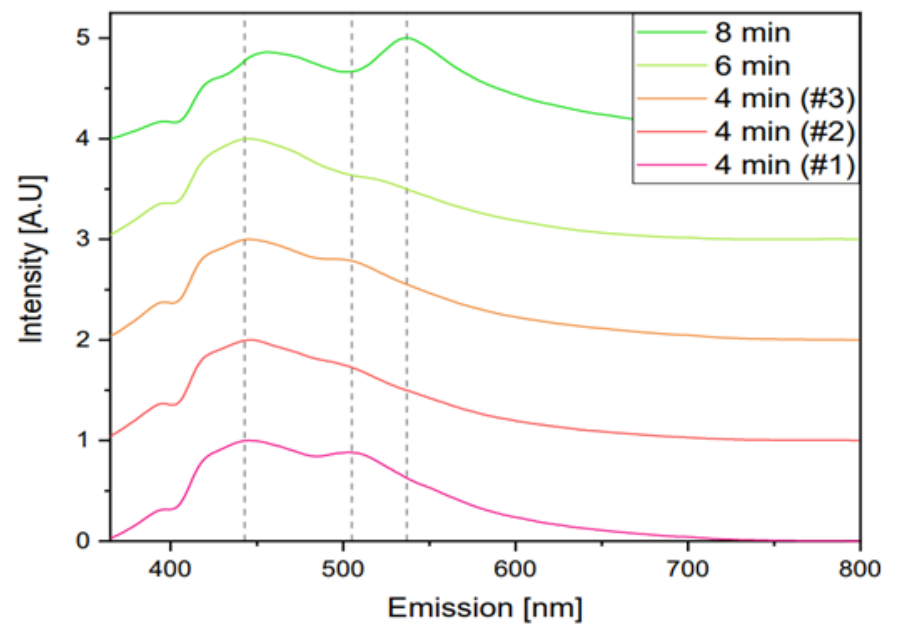

Fig. 14 Microbatch CdSe fluorescence spectra excited at $350 \mathrm{~nm}$ for each peak.

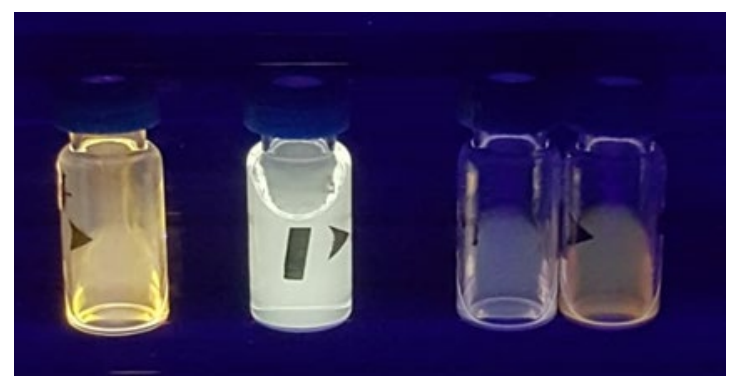

Fig. 15 Emission comparison of CdSe quantum dots. An (a) 4-minute batch sample after two weeks of storage, (b) 4-minute microbatch sample after centrifugation, (c) 4-minute microbatch sample before centrifugation, and (d) an 8-minute microbatch sample.

The PbSe synthesis was performed four times. Once for HR-STEM analysis and three times for IR-fluorescence. The PbSe synthesis was expected to be less efficient than the CdSe synthesis due to the air sensitivity of these quantum dots. Moreover, the microbatch environment forces this synthesis to be a heat-up synthesis rather than the previously used hot-injection, resulting in delayed particle growth and emission at higher energies.

The three IR-fluorescence spectra of 1-minute reaction time were achieved after 18 hours of storage and are shown in Figure 16. Unlike $\mathrm{CdSe}$, the PbSe microbatch experiments showed no clear consistency. Peak emissions at 970, 1,050, and 1,090 nm were retrieved from the analysis, which gave an average emission peak of $1,040 \mathrm{~nm}$ with a standard deviation of $61 \mathrm{~nm}$. This inconsistency in peak emission, however, indicates a stronger particle growth flexibility in one minute reaction time than $\mathrm{CdSe}$ exhibited in six minutes. An increased particle tunability is wanted. Lastly, a PbSe 1-minute batch 
experiment was performed with the remaining precursors, this IR-spectrum is also plotted in Figure 16. This batch experiment did agree with all previous batch experiments and had a peak emission of around $1,160 \mathrm{~nm}$. The PbSe HR-TEM analysis did not yield useful results.

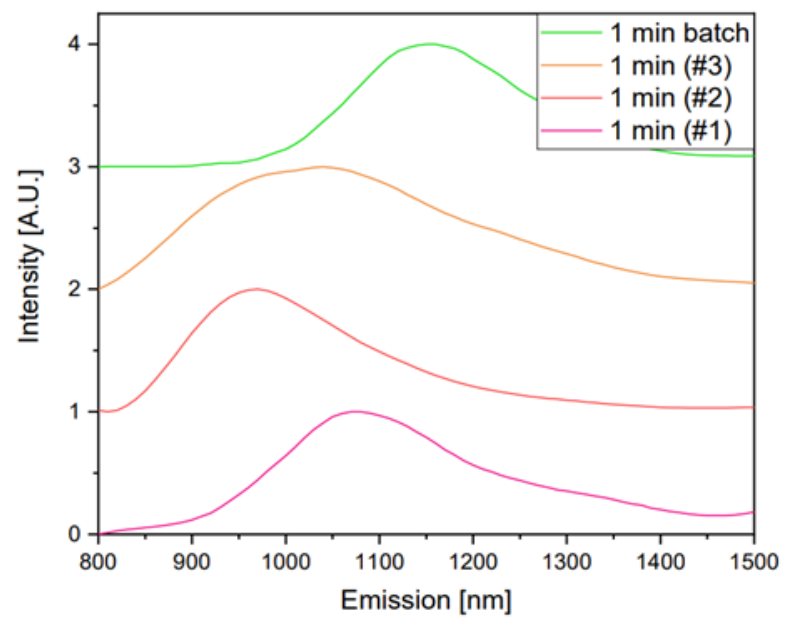

Fig. 16 Microbatch PbSe fluorescence spectra excited at $530 \mathrm{~nm}$. The graph is normalized for each peak.

All microbatch syntheses showed that quantum dots can be formed quickly with a microheater and in microvolumes, the microbatch setup is repeatable for CdSe but shows a strong growth delay. The results are not reproducible for $\mathrm{PbSe}$, yet shows a slow growth delay. The variety in $\mathrm{PbSe}$ peak emissions and lack of CdSe growth can also be (partly) explained by the fact that no active mixing is present: only natural convection arising from a change in fluid temperature and density ensures fluid movement here on Earth, not in space. Therefore, the logical next step is to move to a microflow chip for increased mixing and, of course, space applicability as elaborated later in the Outlook section.

\section{Conclusions}

The results of this study illustrate that quantum dots can be used as a spectral decoy to divert rocket attacks from flying objects such as satellites. We have solved critical issues of a first quantum dot application based on a Raytheon patent aiming for industrial use ${ }^{25}$. Our results revealed that CdSe is emission-tunable, yet unable to reach deep-red to NIR emissions within the strict time limit. The heatup synthesis has shown to be problematic in batch. We suggested switching both the quantum dot material and synthesis approach from the CdSe heat-up method to a PbSe hot-injection synthesis. Switching to PbSe also allowed for rapidly and consistently reaching NIR-emissions. Furthermore, the change from heat-up to hot-injection concepts avoids the nucleation-growth gap experienced in the original heat-up CdSe synthesis.

Both the CdSe and PbSe syntheses were successfully performed in a heat-up microbatch chip. Several technical challenges and issues have been pointed out and partly addressed, which are valuable for a robust design of an autonomous onboard quantum-chip synthesis for the target application. Firstly, the CdSe microsynthesis emission graphs showed no significant change in emission before eight minutes. No significant particle growth occurred in this microbatch reaction environment. Secondly, the slow reaction and insignificant emission in CdSe motivated us to switch to PbSe microsynthesis, which did overcome these issues. However, the PbSe approach was less consistent than batch synthesis. Furthermore, the emission peaks were at a higher-energy wavelength, caused by switching from the hot-injection to the heat-up synthesis. The result thus requires an extended reaction time to reach the wanted lower-energy wavelengths. Lastly, active mixing in such a microbatch environment is imperative for the use in space, as the microgravitational environment present in space does not allow 
for natural convection, which in turn will severely limit the mixing capability and thus repeatability of the chip design. Considering repeatability as an extremely important factor in an autonomous defense mechanism, we proposed a new microchip design in the following outlook section, which marks the next stage of our ongoing developments.

The main outcome of the research reported here is showing how interdisciplinary research, nanomaterial chemistry, exploration of heating and mixing batch microfluidics, and fabrication of miniaturized devices, can enable a stringent, real-life performance under the challenging conditions of the outer space.

\section{Outlook}

An on-board (in-situ) and real-time synthesis of quantum dots is proposed as it was shown that coagulation and aggregation of quantum dots blue-shifts yet narrows the emission spectrum after just 9 days of lab storage, rendering long-term quantum dot storage undesirable. We consider a microfluidic chip for future synthesis because of the capability of fast, compact synthesis. Proper engineering of the exo-atmospheric microchip is necessary to overcome challenges imposed by the predicted operational environment. These challenges include the microgravitational environment, extreme pressure and temperatures, a strict time- and weight limit, and the required chip autonomy. A robust pump-free fluid motion has to be realized as pumps are far too big and heavy objects. Furthermore, the synthesis has to be accomplished in less than $180 \mathrm{~s}$, the time the Indian missile took to reach its satellite target.

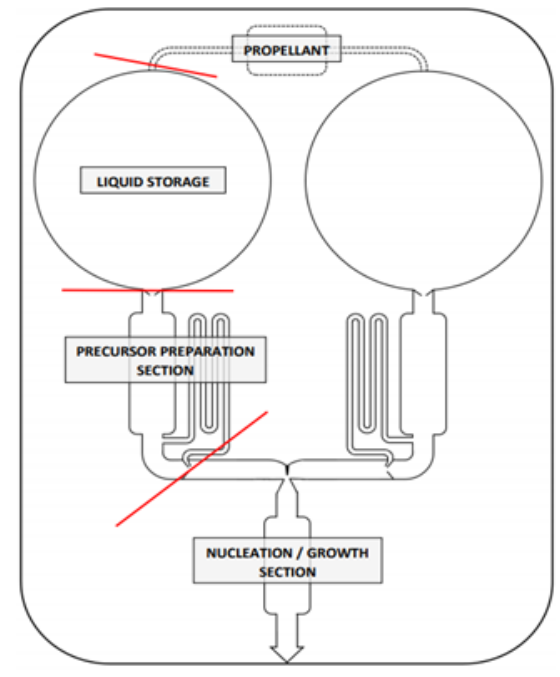

Fig. 17 Initial microfluidic chip design for producing nanodots as a spectral decoy for satellite counter-attack; based on the results of this paper.

The microbatch chip synthesis is finally deemed unsuitable for space and this led to a thinktank evaluation, out of which resulted in a design for a microflow chip. This flow chip should divide the quantum dot preparation into several sections: the liquid/solvent storage, precursor preparation, nucleation/growth, and a propellant section to move the liquids through the chip. The liquid storage section will contain the solvent necessary for the nanocrystal production, here being diphenyl ether + oleic acid, in one compartment and trioctylphosphine in the other compartment. Premade precursor storage should be avoided as the precursors could precipitate. Such a setup is shown in Figure 17.

The proposed design was in part designed by the University of South Australia. The chip was produced at the South Australian Node of the Australian National Fabrication Facility, which is a company established under the National Collaborative Research Infrastructure Strategy to provide micro and nanofabrication facilities for Australia's researchers. 


\section{Acknowledgement}

Volker Hessel acknowledges his start-up fund provided by the Faculty of Engineering, Mathematical \& Computer Sciences and the School of Chemical Engineering and Advanced Materials at The University of Adelaide, Australia.

\section{Reference}

1. Lu, H., Carroll, G. M., Neale, N. R. \& Beard, M. C. Infrared Quantum Dots: Progress, Challenges, and Opportunities. (2019). doi:10.1021/acsnano.8b09815

2. Anderson, S. W., Christensen, K. \& LaManna, J. The development of natural resources in outer space. J. Energy Nat. Resour. Law 37, 227-258 (2019).

3. Packard, K., Goodman, D. \& Whittington, J. NASA Johnson Space Center Small Business Innovation Research (SBIR) Successes, Infusions and Commercializations and Potential International Partnering Opportunities. (2016).

4. Borowski, S. K., Ryan, S. W., McCurdy, D. R. \& Sauls, B. G. Commercialization and Human Settlement of the Moon and Cislunar Space - A Look Ahead at the Possibilities Over the Next 50 Years. in AIAA Propulsion and Energy 2019 Forum (American Institute of Aeronautics and Astronautics, 2019). doi:10.2514/6.2019-3971

5. Van Niekerk, A. F. \& Combrinck, L. The use of civilian-type GPS receivers by the military and their vulnerability to jamming. S. Afr. J. Sci. 108, (2012).

6. Dawson, L. A Summary of the US Space Program and Its Relationship to the Military. in War in Space 61-86 (Springer International Publishing, 2018). doi:10.1007/978-3-319-93052-7_5

7. Voigt, D. The Satellite's Downfall: A Case Study On The Military And Legal Implications Of Chinese Anti- Satellite Missiles. (Leiden University, 2017).

8. Hands, A. D. P., Ryden, K. A., Meredith, N. P., Glauert, S. A. \& Horne, R. B. Radiation Effects on Satellites During Extreme Space Weather Events. Sp. Weather 16, 1216-1226 (2018).

9. Liou, J.-C. \& Krisko, P. An update on the effectiveness of postmission disposal in LEO. (2013). doi:IAC13-A6.4.2

10. Zohuri, B. Directed-Energy Beam Weapons. (Springer, Cham, 2019). doi:10.1007/978-3-030-20794-6

11. Grier, P. The flying tomato. AIR FORCE Magazine 66-68 (2009).

12. Liemer, R. \& Chyba, C. F. A verifiable limited test ban for anti-satellite weapons. Wash. Q. 33, 149-163 (2010).

13. Johnson, N. L. et al. The characteristics and consequences of the break-up of the Fengyun-IC spacecraft. in International Astronautical Federation - 58th International Astronautical Congress 2007 3, 1838-1846 (2007).

14. Pardini, C. \& Anselmo, L. USA-193 decay predictions using public domain trajectory data and assessment of the post-intercept orbital debris cloud. Acta Astronaut. 64, 787-795 (2009).

15. Gertz, B. Russia Flight Tests Anti-Satellite Missile - Washington Free Beacon. (2015). Available at: https://freebeacon.com/national-security/russia-conducts-successful-flight-test-of-anti-satellite-missile/. (Accessed: 14th November 2019)

16. Krishnan, A. Mission Shakti: DRDO's A-SAT missile hit Microsat-R in 168 secs! | National News | English Manorama. (2019). Available at: https:/english.manoramaonline.com/news/nation/2019/03/27/mission-shakti-drdo-asat-missile-hitmicrosat-r-isro.html. (Accessed: 14th November 2019)

17. Precision Weapons | Raytheon. Available at: https://www.raytheon.com/capabilities/precision. (Accessed: 14th November 2019)

18. Titterton, D. H. Development of infrared countermeasure technology and systems. in Mid-infrared semiconductor optoelectronics (ed. Krier, A.) 635-671 (Springer, 2006). doi:10.1007/1-84628-209-8_20

19. Kuwahara, T., Matsuo, S. \& Shinozaki, N. Combustion and sensitivity characteristics of Mg/TF pyrolants. Propellants, Explos. Pyrotech. 22, 198-202 (1997). 
20. Göçmez, A., Yilmaz, G. A., Pekel, F. \& Özkar, S. Development of MTV compositions as igniter for HTPB/AP based composite propellants. Propellants, Explos. Pyrotech. 24, 65-69 (1999).

21. Koch, E. C. Metal-fluorocarbon-pyrolants: III. Development and application of Magnesium/Teflon/Viton (MTV). Propellants, Explos. Pyrotech. 27, 262-266 (2002).

22. Willers, M. S. \& Willers, C. J. Key considerations in infrared simulations of the missile-aircraft engagement. Proc. SPIE 8543, (2012).

23. Abramov, P. I., Kuznetsov, E. V. \& Skvortsov, L. A. Prospects of using quantum-cascade lasers in optoelectronic countermeasure systems: review. J. Opt. Technol. 84, 331 (2017).

24. Huang, H., Tong, Z., Li, T., Jia, L. \& Li, S. Defense Strategy of Aircraft Confronted with IR Guided Missile. Math. Probl. Eng. 2017, (2017).

25. Mintz, N., Skidmore, M. \& Spariosu, K. Countermeasure system and method using quantum dots. (2011).

26. Agrawal, A. et al. Localized Surface Plasmon Resonance in Semiconductor Nanocrystals. (2018). doi:10.1021/acs.chemrev.7b00613

27. Baskoutas, S. \& Terzis, A. F. Size-dependent band gap of colloidal quantum dots. J. Appl. Phys. 99, (2006).

28. Sykora, M. et al. Effect of air exposure on surface properties, electronic structure, and carrier relaxation in PbSe nanocrystals. ACS Nano 4, 2021-2034 (2010).

29. Ramasamy, P., Kim, N., Kang, Y.-S., Ramirez, O. \& Lee, J.-S. Tunable, Bright, and Narrow-Band Luminescence from Colloidal Indium Phosphide Quantum Dots. Chem. Mater. 29, 6893-6899 (2017).

30. Resch-Genger, U., Grabolle, M., Cavaliere-Jaricot, S., Nitschke, R. \& Nann, T. Quantum dots versus organic dyes as fluorescent labels. Nature Methods 5, 763-775 (2008).

31. Zhang, Y. \& Clapp, A. Overview of Stabilizing Ligands for Biocompatible Quantum Dot Nanocrystals. Sensors 11, 11036-11055 (2011).

32. Elbasuney, S. et al. Infrared Spectra of Customized Magnesium/Teflon/Viton Decoy Flares. Combust. Explos. Shock Waves, 55, 93-100 (2019).

33. Kassemi, S. A. \& Ostrach, S. Nature of Buoyancy-Driven Flows in a Reduced-Gravity Environment. AIAA J. 30, 1815-1818 (1992).

34. Grodzka, P. G. \& Bannister, T. C. Natural convection in low-g environments. in AIAA Journal (American Institute of Aeronautics and Astronautics (AIAA), 1974). doi:10.2514/6.1974-156

35. Zapata, E. The State of Play US Space Systems Competitiveness: Prices, Productivity, and Other Measures of Launchers \& Spacecraft. (2017).

36. Kwon, S. G. \& Hyeon, T. Formation Mechanisms of Uniform Nanocrystals via Hot-Injection and HeatUp Methods. Small 7, 2685-2702 (2011).

37. Jia, G., Xu, S. \& Wang, A. Emerging strategies for the synthesis of monodisperse colloidal semiconductor quantum rods. J. Mater. Chem. C 3, 8284-8293 (2015).

38. $\mathrm{Pu}$, C. et al. Synthetic Control of Exciton Behavior in Colloidal Quantum Dots. J. Am. Chem. Soc. 139, 3302-3311 (2017).

39. Wang, K., Lu, Y., Shao, H. \& Luo, G. Heat-Transfer Performance of a Liquid-Liquid Microdispersed System. Ind. Eng. Chem. Res. 47, 9754-9758 (2008).

40. Gómez-Pastora, J., González-Fernández, C., Fallanza, M., Bringas, E. \& Ortiz, I. Flow patterns and mass transfer performance of miscible liquid-liquid flows in various microchannels: Numerical and experimental studies. Chem. Eng. J. 344, 487-497 (2018).

41. Tian, Z. H., Xu, J. H., Wang, Y. J. \& Luo, G. S. Microfluidic synthesis of monodispersed CdSe quantum dots nanocrystals by using mixed fatty amines as ligands. Chem. Eng. J. 285, 20-26 (2016).

42. Visaveliya, N. et al. Micro-flow assisted synthesis of fluorescent polymer nanoparticles with tuned size and surface properties. Nanotechnol. Rev. 5, 259-272 (2016).

43. Kubendhiran, S., Bao, Z., Dave, K. \& Liu, R.-S. Microfluidic Synthesis of Semiconducting Colloidal Quantum Dots and Their Applications. ACS Appl. Nano Mater. 2, 1773-1790 (2019). 
44. Kikkeri, R., Laurino, P., Odedra, A. \& Seeberger, P. H. Synthesis of Carbohydrate-Functionalized Quantum Dots in Microreactors. Angew. Chemie Int. Ed. 49, 2054-2057 (2010).

45. Tan, T. T., Selvan, S. T., Zhao, L., Gao, S. \& Ying, J. Y. Size Control, Shape Evolution, and Silica Coating of Near-Infrared-Emitting PbSe Quantum Dots. (2007). doi:10.1021/cm061974e

46. Phan, H. P. et al. Single-Crystalline 3C-SiC anodically Bonded onto Glass: An Excellent Platform for High-Temperature Electronics and Bioapplications. ACS Appl. Mater. Interfaces 9, 27365-27371 (2017).

47. Dinh, T. et al. An On-Chip SiC MEMS Device with Integrated Heating, Sensing, and Microfluidic Cooling Systems. Adv. Mater. Interfaces 5, 1800764 (2018).

48. Peng, X., Wickham, J. \& Alivisatos, A. P. Kinetics of II-VI and III-V colloidal semiconductor nanocrystal growth: 'Focusing' of size distributions [15]. Journal of the American Chemical Society 120, 5343-5344 (1998).

49. Mokari, T., Sztrum, C. G., Salant, A., Rabani, E. \& Banin, U. Formation of asymmetric one-sided metaltipped semiconductor nanocrystal dots and rods. Nat. Mater. 4, 855-863 (2005).

50. Li, R., Luo, Z. \& Papadimitrakopoulos, F. Redox-Assisted Asymmetric Ostwald Ripening of CdSe Dots to Rods. J. Am. Chem. Soc. 128, 6280-6281 (2006). 